\title{
Magnetic excitations
}

\author{
Stéphane Raymond
}

Laboratoire Magnétisme et Diffraction Neutronique, SPSMS, UMR-E 9001, CEA-INAC/UJF-Grenoble 1, 38054 Grenoble, France

Modern physics provides a framework in order to describe the behavior of many interacting particles as a whole system characterized by a ground state and a spectrum of excited states. The corresponding elementary excitations are a basic ingredient of the description of many physical properties. Their knowledge constitutes the best microscopic understanding of a system and from the initial bricks constituted by the energy spectrum $E_{n}$ and the associated wave-functions $\left|\psi_{n}\right\rangle$, one can calculate expectation value of an observable $A$ : $\left\langle A>=\sum_{n} p_{n}<\psi_{n}|A| \psi_{n}>\right.$ where $p_{n}$ is the probability of occupation of level $n$ at the temperature $T, p_{n}=\frac{1}{Z} \exp \left(-\frac{E_{n}}{k_{B} T}\right)$. Inelastic Neutron Scattering (INS) is a very powerfull probe to measure elementary excitations being either related to the lattice (phonons) or to the magnetism (spin waves, spin fluctuations...). In this lecture, we focus on magnetic excitations starting by recalling basic notions of INS and introducing basic instrumentation. Different kind of excitations are then reviewed: local excitations, dispersive excitations and diffuse modes. These excitations are related to different terms of the Hamiltonian describing the magnetic system: spin-orbit coupling, crystal field potential, exchange interaction.

The bulk of the topic of magnetic excitations is covered by the 1987 review article of Stirling and McEwen [1]. In order to study thoroughly the subject, the reader must refer to classical textbooks by order of difficulty: A. Furrer, J. Mesot and T. Strässle [2], G.L. Squires [3], S.W. Lovesey [4], Y.A. Izyumov and N.A. Chernoplekov [5].

An overview of the different inelastic neutron scattering techniques (three axis, time of flight, spin echo and backscattering) can be found in the book "Neutron and X-ray Spectroscopy" [6]. A book by G. Shirane, S.M. Shapiro and J. Tranquada is devoted to the three axis spectrometer [7]. One can also find practical details (in French) on three axis and time of flight instruments in the JDN16 lecture book $[8,9]$.

\section{INELASTIC NEUTRON SCATTERING, INSTRUMENTATION AND CROSS-SECTIONS}

\subsection{Experimental methods}

The momentum and energy conservation of the neutron scattering process are given by:

$$
\mathbf{Q}=\mathbf{k}_{\mathbf{i}}-\mathbf{k}_{\mathbf{f}}
$$

This is an Open Access article distributed under the terms of the Creative Commons Attribution License 4.0, which permits unrestricted use, distribution, and reproduction in any medium, provided the original work is properly cited. 


\section{Collection SFN}

a)

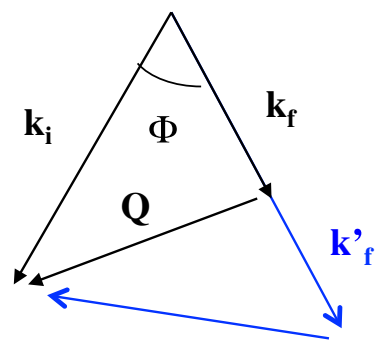

Q' b)

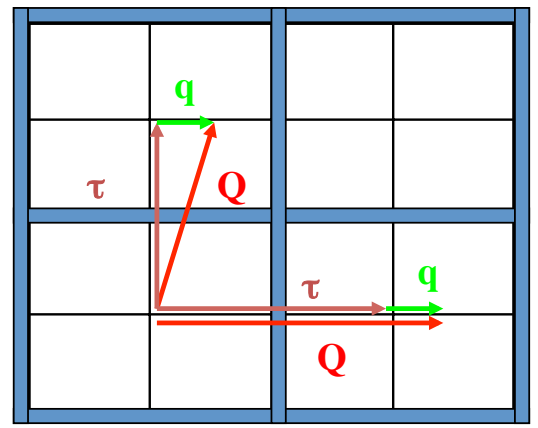

Figure 1. a) Scattering triangle for inelastic neutron scattering. b) Scattering vectors $\mathbf{Q}$ and wave-vectors $\mathbf{q}$ in the reciprocal space of a solid. Blue lines are the Brillouin zone boundaries, $\tau$ vectors are Brillouin zone centers. For the two scattering vectors depicted, the wave-vector $\mathbf{q}$ is the same and lies within the first Brillouin zone. As will be shown latter, the scattering cross-section can be different for these two geometries.

$$
\hbar \omega=E=E_{i}-E_{f}
$$

where the incident neutron of wave-vector $\mathbf{k}_{\mathbf{i}}$ (energy $E_{i}$ ) is scattered into the state with wave-vector $\mathbf{k}_{\mathbf{f}}$ (energy $E_{f}$ ). $\mathbf{Q}$ is the scattering vector and $E=\hbar \omega$ is the energy transfer. The corresponding socalled scattering triangle is depicted in figure 1a. In solids, physics depends only on the relative wavevector defined within a Brillouin zone. Therefore, for inelastic scattering on single crystal sample, $\mathbf{Q}$ is often decomposed into $\mathbf{Q}=\tau+\mathbf{q}$ where $\tau$ is a reciprocal lattice vector and $\mathbf{q}$ is the wave-vector of an elementary excitation to be specified (See Fig. 1b); q lies therefore within the first Brillouin zone. For magnetically ordered systems, one can use the magnetic zone for convenience so that $\mathbf{Q}=\tau+\mathbf{k}+\mathbf{q}$ where $\mathbf{k}$ is the propagation vector of the magnetic structure. For the neutron energy, wave-vector and wavelength, it is useful to have in mind the following relations $E=\frac{h^{2}}{2 m \lambda^{2}}=\frac{\hbar^{2} k^{2}}{2 m}$ ( $m$ is the neutron mass) with the corresponding energy units conversion: $E=81.81 \frac{1}{\lambda^{2}}=2.072 k^{2}(E$ in $\mathrm{meV}, \lambda$ in $\AA$ and $k$ in $\AA^{-1}$ ). Frequency and temperature units are also often used with $1 \mathrm{meV} \sim 0.24 \mathrm{THz} \sim 11.6 \mathrm{~K}$. It is also useful to know that the corresponding energy of a magnetic moment of magnitude $1 \mu_{B}$ in a magnetic field of $1 \mathrm{~T}$ is $0.058 \mathrm{meV}$.

For the system under investigation, the aim of INS is to determine the different energy levels constituting the magnetic excitation spectrum and the possible dispersion relation $E_{n}(\mathbf{q})$ that relates the energy to the wave-vector $\mathbf{q}$ of the elementary excitation. We will treat in this lecture cases where $E_{n}$ does not depends on $\mathbf{q}$ and cases where it does. To have an intuitive picture of the latter case, let's consider a ferromagnetic ground state where all the spins are aligned in a given direction and coupled by an exchange interaction $J$ between nearest neighbors. An excitation at small q (i.e. involving large distances) will be shared between many sites and costs much less energy (down to zero energy for $\mathrm{q}=0$ !) than an excitation at much higher $\mathbf{q}$ involving only neighboring spins that will cost an energy up to $8 J S$ for a ferromagnetic chain. This will be formalized in Section 3.

Figure 1a allows to see a very important feature of neutron scattering: for a given scattering angle $\Phi$, several $\mathbf{Q}$ vectors with different $\mathbf{k}_{\mathbf{f}}$ satisfy the scattering condition (assuming $\mathbf{k}_{\mathbf{i}}$ is fixed for simplicity). There is thus a correlation between the scattering angle, the scattering vector and the energy of the excitation, which is seen from the scattering triangle equation:

$$
\begin{aligned}
Q^{2}=k_{i}^{2}+k_{f}^{2}-2 k_{i} k_{f} \cos \Phi & =k_{i}^{2}\left(2-2 \sqrt{1-\frac{E}{E_{i}}} \cos \Phi-\frac{E}{E_{i}}\right) . \\
\text { 02003-p.2 } & .
\end{aligned}
$$




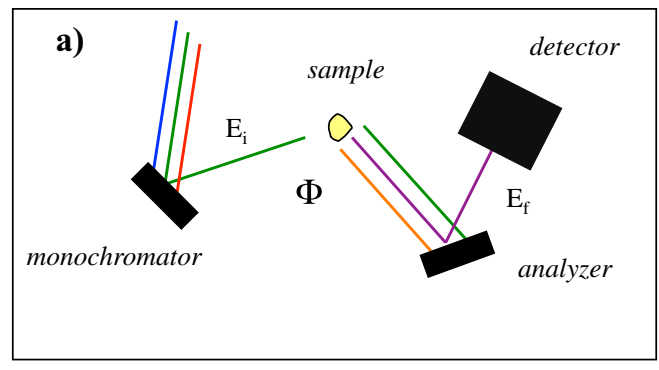

b)

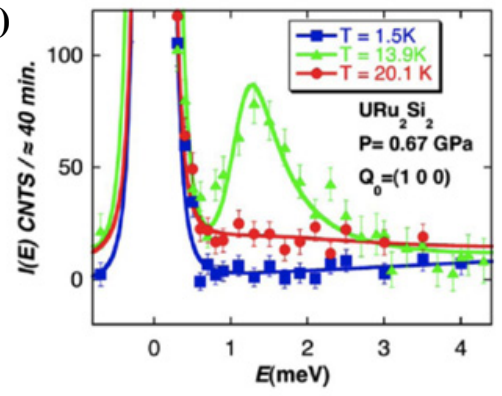

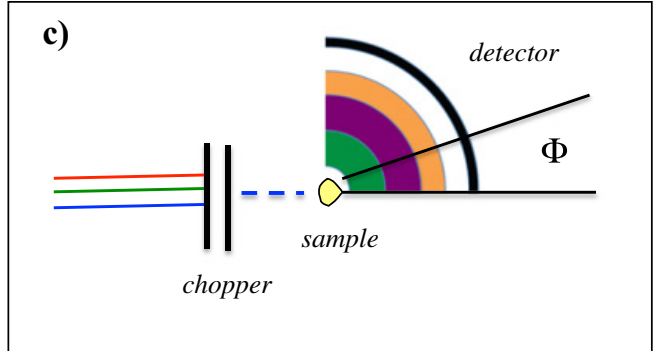

d)

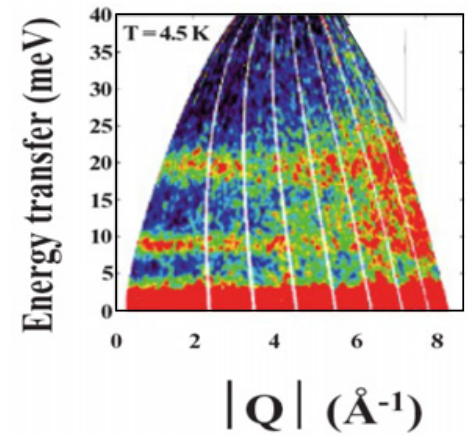

Figure 2. a) TAS principle and b) archetypal spectrum obtained on $\mathrm{URu}_{2} \mathrm{Si}_{2}$ under pressure [10]. A resonance peak only exists for a given $\mathbf{Q}=(1,0,0)$ and a given temperature and pressure. c) TOF principle and d) archetypal spectrum obtained on $\mathrm{CeRhGe}_{3}$ [11]. Well-defined crystal field levels are observed at $7.5 \mathrm{meV}$ and $18 \mathrm{meV}$ at low $Q$ and broad phonon scattering is observed at high $Q$.

The important consequence is as follows: if the sample is a single crystal, for which the data need to be collected for a given $\mathbf{Q}$ vector due to possible anisotropy of physical properties, one needs to rotate the sample in the spectrometer framework in order to satisfy the "scattering triangle condition". For a powder sample, the information on the $\mathbf{Q}$ vector is lost from the sample itself and data are measured for a given scattering angle $\Phi$.

The experimental way of determining the neutron energy leads to different kinds of instruments. One possibility is to use the scattering on a single crystal and the neutron energy (or equivalently wavelength) will be given by the Bragg's law for such a monochromator: $2 d_{M} \sin \theta_{M}=\lambda$ with $d_{M}$ a specific $d$-spacing for a given monochromator (pyrolitic graphite, $\mathrm{Cu}, \mathrm{Si}, \ldots$ ). If the same principle is used to know the final neutron energy (and the device is called an analyzer), the corresponding instrument is a Three Axis Spectrometer (TAS) (the remaining axis being the sample position). Another possibility to measure the neutron energy (or equivalently its speed, $v$ ) is to use the time $t$ taken for traveling through a given distance $L: v=L / t$. This needs a pulsed structure of the beam in order to have a reference of time; it is achieved naturally in pulsed neutron source or one needs to chop the beam in continuous neutron sources. Such a way of determining neutron energy is named Time Of Flight (TOF). A double chopper spectrometer (or TOF-TOF) is an instrument for which both $E_{i}$ and $E_{f}$ are determined by the TOF method. So-called hybrid instruments consist of crystal-TOF or TOF-crystal setups for neutron energy determination. Finally one can make use of the Larmor precession of the neutron spin in a magnetic field $B$, the corresponding angle for a precession in a magnet of length $L$ is $\phi=\gamma B L / v$ where $\gamma=2.916 \mathrm{kHz} / \mathrm{Oe}$ is the Larmor constant. The angle $\Phi$ can be determined by a polarization measurement. This method is used for neutron spin echo instruments that in practice make use of a distribution of wavelengths of about $10 \%$.

Figure 2 pictures TAS and TOF principles and shows corresponding typical spectra. The TAS is better suited for a single crystal sample study since it is built to access a given vector $\mathbf{Q}$. The experiment 


\section{Collection SFN}

is conducted point by point in $(\mathbf{Q}, E)$ space, which matches the "natural" variables $(\mathbf{q}, E)$ of solid state physics. For TOF, large multi-detectors are used and the scattering is simultaneously recorded for many scattering angles $\Phi$. It is thus ideal for powder samples for which $\Phi$ is a "natural" variable. This state of the art is changing due to the increasing mutual trends of using single crystal on TOF (and hence sample orientation and sample rotation stage is needed to access $\mathbf{Q}$ values) and the multiplexing of TAS. Nevertheless one must keep in mind the following rough sketch: TOF are powerful for surveys in a wide $(\mathbf{Q}$, E) space region while TAS are well suited for studying precisely a restricted $(\mathbf{Q}, \mathrm{E})$ region with high neutron flux and a resolution that is easier to tune. Polarized neutron scattering that is very important for magnetism and will be shortly evoked in section 8 is almost solely available on TAS.

To end this brief inelastic neutron scattering instruments overview, we comment the accessible $(\mathbf{Q}, \mathrm{E})$ ranges. The lowest energies are obtained by spin echo and backscattering spectrometers (making use of Bragg scattering in backscattering conditions) while the TAS and TOF cover the remaining of the range. Everything put together INS can be performed in the range $10^{-6}-10^{4} \mathrm{meV}$ and $10^{-4}-20 \AA^{-1}$.

\subsection{Magnetic INS cross-section}

A detailed discussion of the magnetic cross-section for neutron scattering is given in this book by M. Enderle. We recall the magnetic scattering cross-section of unpolarized neutrons for identical magnetic ions with localized and spin-only magnetism:

$$
\frac{d^{2} \sigma}{d \Omega d \omega}=\left(\gamma r_{0}\right)^{2} \frac{k_{f}}{k_{i}} f^{2}(\mathbf{Q}) e^{-2 W(\mathbf{Q})} \sum_{\alpha, \beta}\left[\delta_{\alpha, \beta}-\frac{Q_{\alpha} Q_{\beta}}{Q^{2}}\right] S^{\alpha, \beta}(\mathbf{Q}, \omega) .
$$

Most of the salient features of magnetic neutron scattering are present in this formula:

1 - the scattering amplitude is given by the classical electron radius $r_{0}=0.282 \cdot 10^{-12} \mathrm{~cm}$. It is therefore of the same order of magnitude than the nuclear scattering.

2- the magnetic form factor $f(\mathbf{Q})$ is the Fourier transform of the normalized magnetization density. It indicates that magnetic scattering will be more intense at low $\mathbf{Q}$ (as opposed to phonon scattering).

3- the polarization factor $\left[\delta_{\alpha, \beta}-\frac{Q_{\alpha} Q_{\beta}}{Q^{2}}\right]$ indicates that only components of fluctuations perpendicular to $\mathbf{Q}$ are probed.

$e^{-2 W(\mathbf{Q})}$ is the Debye-Waller factor. Finally the scattering function $S^{\alpha, \beta}(\mathbf{Q}, \omega)$ is the part that will be evaluated in this lecture for several kinds of magnetic excitations. We will see that depending on the nature of the excitation, one of the following description of the scattering function is more adequate:

A- in term of matrix elements: $<\Gamma_{n}\left|\widehat{S}_{i}^{\alpha}\right| \Gamma_{m}><\Gamma_{m}\left|\widehat{S}_{j}^{\beta}\right| \Gamma_{n}>$ (where $\widehat{S}^{\alpha}$ is the component $\alpha$ of the spin operator at site $i$ ).

B- in term of spin-spin correlation function: $<\widehat{S}_{i}^{\alpha}(0) \widehat{S}_{j}^{\beta}(t)>$.

C- in term of the imaginary part of the dynamical spin susceptibility: $\chi^{\prime \prime \alpha, \beta}(\mathbf{Q}, \omega)$.

In the latter case, the scattering function is related to the imaginary part of the of the dynamical spin susceptibility by the fluctuation-dissipation theorem ${ }^{1}$ :

$$
S^{\alpha, \beta}(\mathbf{Q}, \omega)=\frac{1}{1-\exp \left(-\hbar \omega / k_{B} T\right)} \chi_{s}^{\prime \prime \alpha, \beta}(\mathbf{Q}, \omega) .
$$

\section{LOCAL EXCITATIONS}

In this section, we consider magnetic excitations that concern only a single site $i$ of the magnetic lattice. Typical such excitations are crystal field transitions and spin-orbit transitions. We will focus on crystal

\footnotetext{
1 The subscript $s$ indicates that the symmetric part of the tensor must be considered.
} 
field excitations that are canonical examples of such local excitations. The addition of interactions between sites in crystal field dominated systems leads to the so-called crystal field exciton and this will be treated in Section 4. When there is no correlation between sites $i$ and $j$, the physics is $\mathbf{Q}$ independent in reciprocal space (This is illustrated in Fig. $2 \mathrm{~d}$ for the crystal field levels of $\mathrm{CeRhGe}_{3}$ ). However, the measured neutron intensity may be $\mathbf{Q}$ dependent due to the magnetic form factor, the polarization factor and the $\alpha$ dependence in the scattering function (See Eq. (1.4)).

Crystal field interaction corresponds to the electrostatic interaction between a magnetic ion and its neighboring ions. Since the resulting local symmetry is lower than the spherical symmetry of the "free" magnetic ion, a degeneracy lifting occurs. A very common example is the splitting in $t_{2 g}$ and $e_{g}$ levels for $d$ orbital. Crystal field is very important for rare-earth ( $4 f$ electron) compounds since it is usually weaker than spin-orbit coupling (typical crystal field energies span the range $0.1-1 \mathrm{eV}$ for $d$ electron systems and 10-100 meV for $f$ electron systems). We restrict the following discussion to crystal field in $f$ electron systems. For a crystal field transition in $4 f$ electron systems for which the total angular momentum $J$ is a good quantum number, the direct use of matrix element is convenient and the neutron scattering cross-section writes:

$\frac{d^{2} \sigma}{d \Omega d \omega}=N\left(\gamma r_{0}\right)^{2} \frac{k_{f}}{k_{i}} f^{2}(\mathbf{Q}) e^{-2 W(\mathbf{Q})} \sum_{\alpha, n, m} p_{n}\left[1-\left(\frac{Q_{\alpha}}{Q}\right)^{2}\right]\left|<\Gamma_{m}\right| \widehat{J}^{\alpha}\left|\Gamma_{n}>\right|^{2} \delta\left(\hbar \omega+E_{n}-E_{m}\right)$.

In this formula, $p_{n}$ is the Boltzmann population of the level $\Gamma_{n}$ and $N$ is the number of identical magnetic ions in the system. It is to be noted that the matrix elements can lead to some $\mathbf{Q}$ dependence of the scattering since they may have different values for the transverse components $\alpha=x, y$ and the longitudinal one $\alpha=z$. Here we are in a case where everything can be in principle calculated and the neutron intensity will give information on the matrix elements and consequently the eigenvectors of the problem and the peak position will give the eigenenergies. The crystal field Hamiltonian writes easily by symmetry considerations when considering the neighboring ions as point charges:

$$
H_{C E F}=-e V(\mathbf{r})=\sum_{m, n} B_{n}^{m} \widehat{O_{n}^{m}} .
$$

In the first hand of Eq. (2.2), one considers a general writing in term of an aspherical potential $V(\mathbf{r})$ invariant under symmetry of the considered crystallographic site. It can be expressed using spherical harmonics expansion and can be expressed in term of $J$ operators for rare-earth. In this latter case, a very common formalism introduced by Stevens is used and is written in the second hand of Eq. (2.2) where $B_{n}^{m}$ are called the crystal field parameters and $\widehat{O_{n}^{m}}$ are the Stevens operators. For example, in certain amorphous rare earth based compounds, the local axial crystal field Hamiltonian is:

$$
H_{C E F}=B_{2}^{0} \widehat{O_{2}^{0}} \text {. }
$$

This corresponds to a potential that is of the form $3 z^{2}-r^{2}$ in cartesian coordinates. In solids, the Hamiltonian (2.2) contains many terms, it is of sixth order in $n$ for $4 f$ electrons and if a $p$-fold axis of symmetry is chosen as quantification axis, (2.2) is reduced to terms up to $m=p$. The corresponding crystal field states obtained by diagonalizing the Hamiltonian are linear combinations of $\mid J, M>$ states:

$$
\left|\Gamma_{n}>=\sum_{M=-J}^{J} a_{M}\right| J, M>
$$

The compound $\mathrm{NdPd}_{3}$ is taken as an example for crystal field excitation $[2,12] . \mathrm{NdPd}_{3}$ crystallizes in the cubic $\mathrm{AuCu}_{3}$ structure and the $J=9 / 2$ state of free $\mathrm{Nd}^{3+}$ ion is split in two quartets and one doublet in such an environment. The analysis of both the peaks position and the intensities of the INS transitions allows to get the crystal field level scheme $[2,12]$. This leads to a $\Gamma_{8}^{2}$ quartet ground state separated from 


\section{Collection SFN}
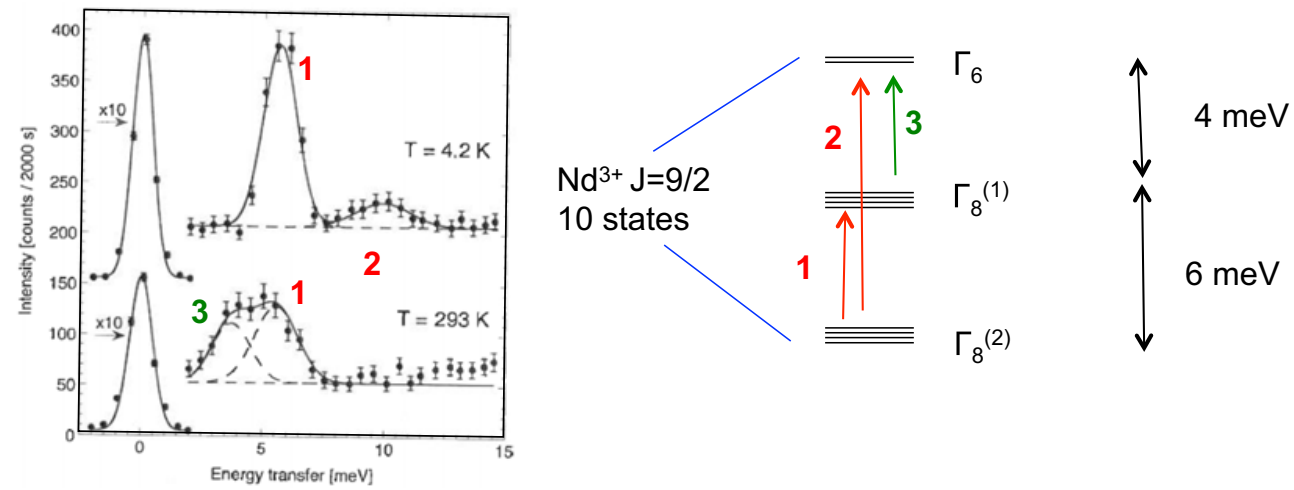

Figure 3. Crystal field transitions measured by INS on powder sample of $\mathrm{NdPd}_{3}$ and corresponding energy levels $[2,12]$. Transitions 1 and 2 correspond to excitations from the ground state to the excited state while transition 3 is an excitation between the thermally populated first excited state and the higher energy second excited state.

a second quartet $\left(\Gamma_{8}^{1}\right)$ at $6 \mathrm{meV}$ and the doublet $\left(\Gamma_{6}\right)$ is separated from the ground state by $10 \mathrm{meV}$ (See Fig. 3 right). At low temperatures (Fig. 3 left), transitions 1 and 2 arise from the ground state to each excited state. At $293 \mathrm{~K}$, a transition between excited states (transition 3) is possible due to the thermal population of the second quartet $\Gamma_{8}^{1}$. This illustrates a very important point concerning the role of the population factor in producing "new" magnetic transitions at high temperature and the counterintuitive fact that magnetic excitations are not necessarily of higher intensity at lower temperature.

The equivalent to crystal field transition of $f$ electrons are usually named $d$ - $d$ excitations for $d$ electron systems. For an example, the reader can look at the recent work performed in $\mathrm{NiO}$ to investigate the $t_{2 g}-e_{g}$ splitting of $1.05 \mathrm{eV}$ [13]. Conversely high energy transitions involving $f$ electrons are intermultiplet transitions, that is transitions between different $J$ levels (e.g. $J \rightarrow J+1$ ). This reflects the usual opposite hierarchy of interaction between spin-orbit and crystal field in rare-earth and transition metal compounds.

Previously we dealt with single ion excitations. Another common case of $\mathbf{Q}$-independent excitations arises in magnetic clusters, that is an assembly of a few interacting magnetic ions. The simplest case is the one of dimers, for which an Heisenberg Hamiltonian couples two (identical) spins 1 and 2: $H=-2 J \mathbf{S}_{1} \cdot \mathbf{S}_{2}$. The total spin $\mathbf{S}=\mathbf{S}_{1}+\mathbf{S}_{2}$ is a good quantum number and the wave-function of the dimer state is therefore described by $\mid S, M>$ with corresponding eigenvalues $E(S)=-J(S(S+1)-$ $\left.2 S_{1}\left(S_{1}+1\right)\right)\left(0 \leq S \leq 2 S_{1}\right)$. Compared to crystal field transition, there is a supplementary structure factor oscillating in $\mathbf{Q}$ space that allows to identify such an excitation. To help understanding, one can still see this kind of excitation as a local one but this time the entity is a dimer instead of a single ion. In the same way, one can built magnetic clusters of many spins that are important in the field of molecular magnetism.

\section{DISPERSIVE EXCITATIONS: SPIN WAVES}

We now consider the exact opposite limit of the previous section in which only local physics was considered. We take the dominant effect in the Hamiltonian to be the exchange interaction between many bodies:

$$
H=-\sum_{i, j} J_{i, j} \mathbf{S}_{i} \cdot \mathbf{S}_{j}=-\sum_{i>j} 2 J_{i, j} \mathbf{S}_{i} \cdot \mathbf{S}_{j} .
$$


One of the most important effect of exchange as concern spin dynamics is the appearance of collective magnetic excitation in ordered magnets below the transition temperature that are called spin waves. In systems with continuous broken symmetry at the ordering transition, the long wavelength spin waves are the modes restoring the broken symmetry equivalently to shear sound for the liquid-solid transition for which the three-dimensional translation symmetry is broken. Typical exchange energy is in the range 1-10 meV for $f$ electron systems and $10-100 \mathrm{meV}$ for $d$ electron systems.

\subsection{A simple start: Ferromagnetic spin waves}

To grasp the physics under the spin wave excitations, let us consider a ferromagnetic compound with a ground state $\Phi$ for which the projection of the magnetic moment along the quantification axis $z$ has its maximum value $S$ for all magnetic sites. Let's then consider an excited state where the site $j$ has a reduced magnetic moment projection from $S$ to $S-1:\left|j>=S_{j}^{-}\right| \Phi>$. It is easy to show that this is not an eigenstate of the Heisenberg Hamiltonian. In contrast, an eigenstate can be built by considering a linear combination of all such $\mid j>$ states: $\left|\mathbf{q}>=\sum_{j} \exp \left(i \mathbf{q} \cdot \mathbf{R}_{j}\right)\right| j>$. This means that the spin deviation from $S$ to $S-1$ is delocalized in space: it cost less energy to share a spin flip between all the sites. This excitation is a spin wave of wave-vector q. The fact that q-space is the "pertinent" one is to be related to the fact that a Fourier transform diagonalizes a quadratic form. In q-space the Heisenberg Hamiltonian writes as:

$$
H=-\sum_{\mathbf{q}} J_{-\mathbf{q}} \mathbf{S}_{\mathbf{q}} \cdot \mathbf{S}_{-\mathbf{q}}
$$

with $J_{-\mathbf{q}}=\sum_{i \neq j} J_{i, j} e^{i \mathbf{q}\left(\mathbf{R}_{i}-\mathbf{R}_{j}\right)}$ and $\mathbf{S}_{\mathbf{q}}=\sum_{i} \mathbf{S}_{i} e^{-i \mathbf{q} \cdot \mathbf{R}_{i}}$. This form is simpler than (3.1) but the commutation relations of $\mathbf{S}_{\mathbf{q}}$ are more complex than the ones of $\mathbf{S}_{i}$ operators. The treatment of spin waves is made in many solid state physics textbooks. It consists in describing the spin operators in terms of bosons and then to diagonalize the Hamiltonian. The corresponding field (or quasiparticle) is called magnon. If $a_{i}^{\dagger}$ is the magnon creation operator at the $i$ site and the corresponding number of magnon is therefore $n_{i}=a_{i}^{\dagger} a_{i}$, we have the following expressions obtained by linearization in $\left.a_{i}: S_{i}^{+} \approx \sqrt{(} 2 S\right) a_{i}$ and $S_{i}^{z}=S_{i}-n_{i}$ which are valid at low temperature when the average value of spin deviation is small $\left(n_{i} \leq 2 S\right)$ and for large spin $S$ ("classical spin"). Within this approximation an ensemble of independent harmonic oscillators is obtained:

$$
H=\sum_{\mathbf{q}} \hbar \omega_{\mathbf{q}} a_{\mathbf{q}}^{\dagger} a_{\mathbf{q}}
$$

where $a_{\mathbf{q}}$ is the Fourier transform of $a_{i}$. The associated dispersion relation is:

$$
\hbar \omega_{\mathbf{q}}=2 S\left[J_{0}-J_{\mathbf{q}}\right] .
$$

For a spin chain with a ferromagnetic exchange $J$ between nearest neighbors distant from $a$, we have:

$$
\hbar \omega_{q}=4 S J[1-\cos (q a)] .
$$

The corresponding dispersion relation is shown in Figure 4 with the physical picture for spin waves: it corresponds to spin precession around the magnetization direction. At low $q$, the dispersion is quadratic: $\hbar \omega_{q}=2 S J a^{2} q^{2}$. The Hamiltonian (3.1) is isotropic. If we add a single ion anisotropy like $-D_{z} S_{z}^{2}$ and consider an easy axis anisotropy $\left(D_{z}>0\right)$, the spin waves spectrum acquires a gap and the dispersion becomes:

$$
\hbar \omega_{\mathbf{q}}=2 S\left[J_{0}-J_{\mathbf{q}}+D_{z}\right] .
$$

This gap at $q=0$ expresses the fact that there is a cost in energy to produce a spin deviation perpendicular to the easy axis. 


\section{Collection SFN}

a)

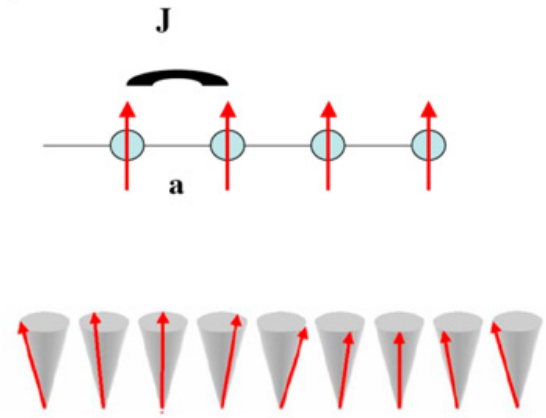

b)

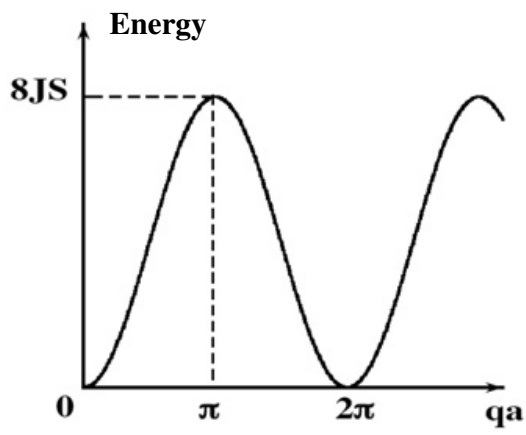

Figure 4. a) Classical picture of a spin wave: spin precession in the ferromagnetic spin chain. b) Dispersion relation of the ferromagnetic spin chain.

After the spin wave energy, the neutron scattering cross-section can be evaluated and the more convenient way is to introduce the spin representation into the spin-spin correlation function; the obtained expression will be touched in the next section.

\subsection{Overview of the more general case}

The general case of spin wave calculation must be performed for magnetic structures having several magnetic ions per magnetic unit cell and considering different kinds of anisotropies: single ion anisotropy, exchange anisotropy. Such a treatments is made in the book of Y.A Izyumov and N.A. Chernoplekov [5] and also in the JDN16 lecture book by S. Petit [14]. The basic idea is to match the well-known method established for the ferromagnetic case. To this aim, a local coordinate frame for each magnetic ion of the magnetic unit cell is introduced and the $z$-axis of the local frame is taken along the ordered moment direction. The bosonization is made in the local frame and the transformation matrices to the cartesian space are defined by the magnetic structure. The expressions for the magnon dispersion relation and the neutron cross-section can then be quite elaborated. One must know that if there are $n$ magnetic atoms per magnetic unit cell, there will be $n$ magnon branches.

For the case of incommensurate magnetic structures, the (quasi)-momentum $\mathbf{q}$ defined in the paramagnetic space group is not conserved. The scheme exposed above still applies but the transformation matrix must be expanded in a Fourier series as a function of atomic position. A simple case is the one of the magnetic spiral with the magnetic moment perpendicular to the propagation vector $\mathbf{k}$. In such a case three vectors are involved in the aforementioned Fourier expansion $(0, \mathbf{k}$ and $-\mathbf{k})$ and spin wave modes are observed at $\hbar \omega_{\mathbf{q}}, \hbar \omega_{\mathbf{q}-\mathbf{k}}$ and $\hbar \omega_{\mathbf{q}+\mathbf{k}}$. For sine-wave modulated structures (the magnitude of the ordered moment changes from site to site), the situation is certainly more complex and the magnetic excitation spectrum can show both sharp dispersive excitations and broad modes.

We now give the salient features of the spin wave neutron cross-section. To this purpose, we go back to a simpler case where all the ordered moments point along the $z$-axis of the cartesian space. In this case the spin wave scattering function $S_{S W}(\mathbf{Q}, \omega)$ is defined by:

$$
\sum_{\alpha, \beta}\left(\delta_{\alpha, \beta}-\frac{Q_{\alpha} Q_{\beta}}{Q^{2}}\right) S(\mathbf{Q}, \omega)=\frac{1}{2}\left(1+\left(\frac{Q_{z}}{Q}\right)^{2}\right) S_{S W}(\mathbf{Q}, \omega) .
$$


a) $\mathrm{MnF}_{2}$

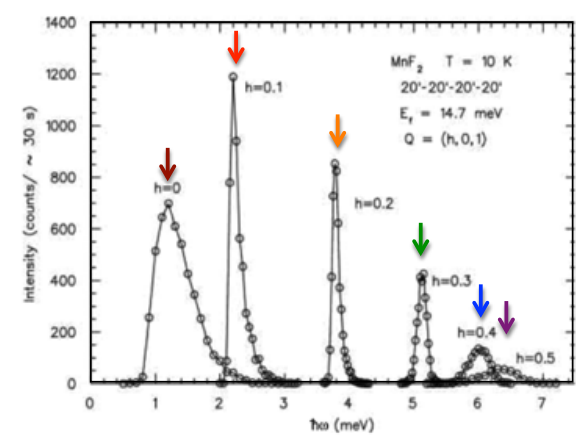

b) $\mathrm{BaNi}_{2}\left(\mathrm{PO}_{4}\right)_{2}$

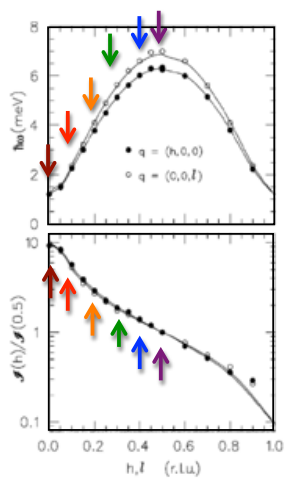

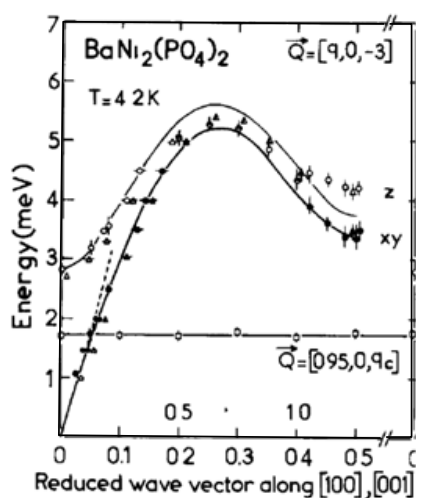

Figure 5. a) Spin wave spectra measured with a TAS on $\mathrm{MnF}_{2}$ with the corresponding dispersion relation and normalized intensities [7, 15]. b) Spin wave dispersion in $\mathrm{BaNi}_{2}\left(\mathrm{PO}_{4}\right)_{2}$ along $[1,0,0]$ and $[0,0,1][16]$.

The maximum intensity is obtained for $\mathbf{Q} / / \mathrm{z}$ since the magnetic moments are along $z$ and spin waves are transverse motions (note that the magnetic elastic intensity has zero intensity for such a configuration). The spin-spin correlation function can be expressed in the boson operator framework and this leads to the following spin wave scattering function:

$$
S_{S W}(\mathbf{Q}, \omega)=S \sum_{\mathbf{k}, \mathbf{q}} t^{2}(\mathbf{Q})\left[\left(n_{\mathbf{q}}+1\right) \delta(\mathbf{Q}-\mathbf{q}-\mathbf{k}) \delta\left(\omega-\omega_{\mathbf{q}}\right)+n_{\mathbf{q}} \delta(\mathbf{Q}+\mathbf{q}-\mathbf{k}) \delta\left(\omega+\omega_{\mathbf{q}}\right)\right] .
$$

First the intensity is proportional to $S$ and not to the square of the ordered moment like the intensity of magnetic Bragg scattering (the total scattered magnetic intensity integrated all over $\mathbf{Q}$ and $\omega$ must be proportional to $S(S+1)$ ). Second there is a structure factor $t(\mathbf{Q})$; it is 1 for a ferromagnet and $2 z J S / \hbar \omega_{\mathbf{q}}$ for a simple antiferromagnet at low $\mathbf{q}$ for which $J$ is the exchange integral between $z$ nearest neighbors. For such a simple antiferromagnet, the dispersion is linear at small $q: \hbar \omega_{q}=z J S a q$ (instead of quadratic for the Heisenberg ferromagnet with nearest neighbor interactions) and hence the intensity decreases when $q$ increases from the antiferromagnetic zone center (at small $q$ ). The other factors indicate that spin waves give rise to sharp peaks in $\mathbf{Q}$ and $\omega$ space. However spin waves acquire a finite lifetime when further interactions are taken into account e.g. magnon-magnon scattering.

\subsection{Examples}

The basic concepts exposed above are illustrated in this section with two examples. Figure 5a shows the spin wave measurements performed on the "textbook" antiferromagnetic compound $\mathrm{MnF}_{2}$ [7, 15]. The left panels shows the spectrum collected at $T=10 \mathrm{~K}\left(T_{N}=67.5 \mathrm{~K}\right)$ along $(h, 0,1)$. The TAS is setup to collect a spectrum for a single $h$ value along $(h, 0,1)$. The peak positions (arrows) obtained for each $h$ are reported on the dispersion relation shown in the right panel (top). One can notice that the measured peaks are sharp except when the dispersion flattens (at the zone center and zone border). This is related to focusing effect associated with the TAS resolution: it is possible to match the shape of the resolution function and the dispersion when this latter is almost linear (see [7] for more details). The bottom right panel shows the normalized intensity associated with the dispersion relation. Due to the factor $t^{2}(\mathbf{Q})$, the intensity does not follow the periodicity of the dispersion and decreases continuously being maximum at the antiferromagnetic Bragg peak position and reaching zero when the antiferromagnetic peak structure factor vanishes. 


\section{Collection SFN}

Figure $5 \mathrm{~b}$ shows the dispersion relation obtained for the honeycomb lattice $\mathrm{S}=1$ antiferromagnetic compound $\mathrm{BaNi}_{2}\left(\mathrm{PO}_{4}\right)_{2}$ [16]. The honeycomb lattice can be decomposed in two hexagonal Bravais lattices leading thus to two magnetic atoms per unit cell. It follows that two branches of spin waves are observed: an acoustic branch with a linear dispersion at low $q$ and an optic branch with a gap at $q=0$. This gap reflects the strong planar anisotropy of the system while the absence of gap for the other mode reflects the absence of sizable in-plane anisotropy. Along the $c$-axis the dispersion is flat indicating the two dimensionality of the system.

\section{CRYSTAL FIELD EXCITON}

In the previous sections, we treated first the local excitations with the example of crystal field (Sect. 2) and then the spin waves in ordered magnets arising from the exchange interaction (Sect. 3). In this section we want to have some insight on systems for which both terms of the Hamiltonian are of the same order of magnitude. This arises for example for exchange energies and crystal field potentials in some rare earth compounds. A very convenient way to integrate several such terms of the Hamiltonian is to use the Random Phase Approximation (RPA). If $\chi_{0}$ is the susceptibility of the non interacting system, the inclusion of interaction $J_{\mathbf{q}}$ leads to the RPA susceptibility:

$$
\chi(\mathbf{q}, \omega)=\frac{\chi_{0}(\mathbf{q}, \omega)}{1-J_{\mathbf{q}} \chi_{0}(\mathbf{q}, \omega)} .
$$

From this formula, it is clear that interactions may enhance the susceptibility as compared to the bare one for certain wave-vectors.

We now illustrate this for a praseodymium based compound. In $\mathrm{PrOs}_{4} \mathrm{Sb}_{12}$, the $4 f^{2}$ configuration of $\operatorname{Pr}^{3+}(J=4)$ is split by the crystal field potential into one singlet and three doublets. The ground state is the singlet $\Gamma_{1}$ and the first excited state is the triplet $\Gamma_{4}^{2}$ at an energy $\Delta \approx 0.7 \mathrm{meV}$. The local crystal field susceptibility writes:

$$
\chi_{0}(\omega)=\left(f_{0}-f_{1}\right) \frac{\Delta M^{2}}{\Delta^{2}-(\hbar \omega+i \epsilon)^{2}}
$$

where $f_{0}$ is the thermal population of the ground state and $f_{1}$, the one of the excited state. $M$ is the dipolar matrix element between the two levels: $M=\left\langle\Gamma_{4}^{2}\left|J_{x}\right| \Gamma_{1}\right\rangle$ and $\epsilon \rightarrow 0$. This susceptibility is q-independent since the crystal field potential acts on a single site $(i)$, the one of a single Pr ion. Including exchange interaction interaction $I(\mathbf{q})$ between sites and using the RPA Eq. (4.1) leads to:

$$
\chi(\mathbf{q}, \omega)=\frac{\left(f_{0}-f_{1}\right) \Delta M^{2}}{\Delta^{2}-(\hbar \omega+i \epsilon)^{2}-I(\mathbf{q})\left(f_{0}-f_{1}\right) \Delta M^{2}}
$$

with the dispersion relation of the excitation given by the pole of Eq. (4.3):

$$
\hbar \omega_{\mathbf{q}}=\sqrt{\Delta\left(\Delta+\left(f_{0}-f_{1}\right) M^{2} I(\mathbf{q})\right)}
$$

Such a mode corresponds to a crystal field excitation propagating from site to site; it is usually called crystal field exciton. The interaction gives rise to a modulation of the crystal field level in $\mathbf{q}$-space for $T \ll \Delta$ (where $f_{0}=1$ and $f_{1}=0$ ). A sketch of this excitation is given in the left panel of Figure 6 while the right panel shows the excitation spectrum measured by INS along $[1,0,0]$ in $\mathrm{PrOs}_{4} \mathrm{Sb}_{12}$ : the crystal field splitting of $0.7 \mathrm{meV}$ is renormalized by interaction and acquires a dispersions [17].

\section{DIFFUSE MODES FOR LOCAL MOMENTS}

In the previous sections, we dealt with well defined excitations for which the magnetic susceptibility has a pole that corresponds to an eigenenergy of the system (and eigenvectors are involved in the 
$\Delta$

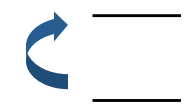

Site i

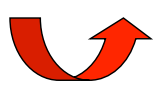

Interaction : I

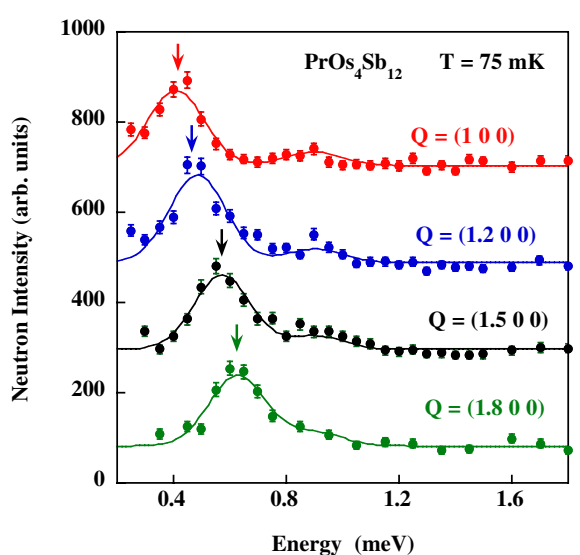

Figure 6. Left: sketch of the crystal field exciton: it is a crystal field excitation propagating from site to site due to the exchange interaction. Right: magnetic excitation spectrum measured in $\mathrm{PrOs}_{4} \mathrm{Sb}_{12}$ [17].

matrix elements giving the INS intensity). We will now consider the so-called diffuse excitations that are broad in $(\mathbf{q}, \omega)$ space. The most common case, for localized spin systems, is the paramagnetic scattering occurring above the ordering temperature. In the paramagnetic phase, the spins see an effective fluctuating field which leads to finite spin-spin correlations. These correlations increase when $T$ approaches the transition temperature leading eventually to long range magnetic order. In the following, we consider a simple case that applies to certain class of compounds. The instantaneous spin correlation function is postulated to be of the same type as that assumed in the Ornstein-Zernike theory of critical opalescence (for simplicity we do not consider anisotropy):

$$
<S_{0}^{\alpha}(0) S_{R}^{\alpha}(0)>\propto \frac{e^{-\kappa R}}{R}
$$

where $\kappa=1 / \xi$ is the inverse correlation length. The time dependence is usually taken into account by making the relaxation rate hypothesis:

$$
<S^{\alpha}(q, 0) S^{\alpha}(q, t)>=<S^{\alpha}(q, 0) S^{\alpha}(q, 0)>e^{-\left(\Gamma_{q} / \hbar\right) t}
$$

where $\Gamma_{q}$ is a $q$ dependent relaxation rate. The scattering cross-section becomes:

$$
S(\mathbf{Q}, \omega)=\frac{2 k_{B} T \chi(0)}{\pi} \frac{\kappa^{2}}{\kappa^{2}+q^{2}} \frac{\Gamma_{q}}{(\hbar \omega)^{2}+\Gamma_{q}^{2}}
$$

where $\chi(0)$ is the static susceptibility. This expression is valid at high temperature only due to the approximations made in the microscopic model leading to (5.2). On another hand, the following scattering function is useful to describe the data for all temperatures:

$$
S(\mathbf{Q}, \omega)=\frac{\hbar \omega / k_{B} T}{1-\exp \left(-\hbar \omega / k_{B} T\right)} \frac{2 k_{B} T \chi(0)}{\pi} \frac{\kappa^{2}}{\kappa^{2}+q^{2}} \frac{\Gamma_{q}}{(\hbar \omega)^{2}+\Gamma_{q}^{2}} .
$$

For a ferromagnet, diffusion theory predicts $\Gamma_{q}=\Lambda q^{2}$ where $\Lambda$ is the diffusion constant. Figure 7 shows typical spectra obtained above the Curie temperature of the ferromagnetic compound $\mathrm{UGe}_{2}$ [18]. When $q$ increases from the ferromagnetic position $(0,0,1)$, the spectrum gets broader due to the fact that $\Gamma_{q}$ increases with $q$. Indeed, the data are collected for a regime where $k_{B} T>>\hbar \omega$ so that an inspection of Eq. (5.4) indicates that the half width at half maximum of the peak is proportional to $\Gamma_{q}$. 


\section{Collection SFN}
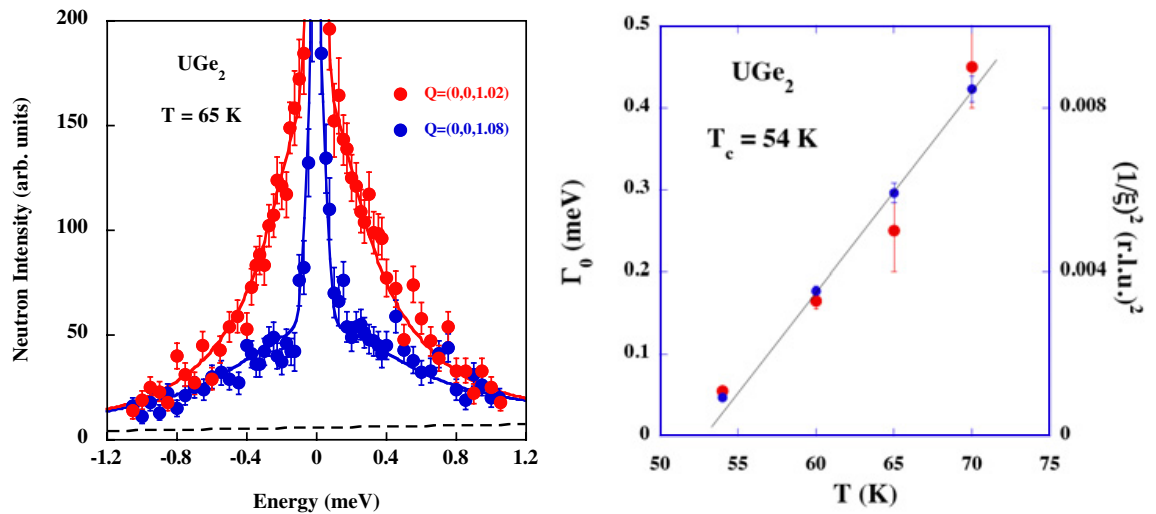

Figure 7. Left: excitation spectrum measured in $\mathrm{UGe}_{2}$ above $T_{\text {Curie }}$. Right: temperature dependence of $\Gamma_{0}$ and $1 / \xi^{2}$ adapted from Ref. [18].

The right panels shows the temperature variation of $\Gamma_{0}$ and $1 / \xi^{2}$. It follows that $\Gamma_{0} \propto 1 / \xi^{2}$. This indicates that when the correlation length increases on approaching $T_{c}$, the relaxation rate decreases: this is the so-called slowing down of fluctuations. At $T_{c}, \Gamma_{0} \rightarrow 0$ and $\xi \rightarrow \infty$.

We will end this section by another example of relaxation for which there is no phase transition occurring at low temperature so that the spectrum has always the same broad features down to $T=0 \mathrm{~K}$. This allows to illustrate the peculiar shape of $\chi^{\prime \prime}(\mathbf{Q}, \omega)$ and $S(\mathbf{Q}, \omega)$ at low temperature (as opposed to the regime studied just above where $\left.k_{B} T>>\hbar \omega\right)$. The example is the one of a localized magnetic moment embedded in a conduction electron sea. For such systems, the local magnetic moment (on site $i$ ) is screened by the conduction electron via the so-called Kondo interaction. For the case where there is no interaction between sites $i$ and $j$ (for example in dilute alloys), there is no q dependence in the phenomenon and the local spin relaxation will be described by a relaxation rate $\Gamma_{K}$ and the corresponding imaginary part of the dynamical susceptibility is:

$$
\chi^{\prime \prime}(\omega)=\chi_{K} \frac{\omega \Gamma_{K}}{\omega^{2}+\Gamma_{K}^{2}}
$$

while the scattering function is given by the fluctuation-dissipation theorem Eq. (1.5).

Figure 8 shows the scattering function measured in $\mathrm{CeRu}_{2} \mathrm{Si}_{2}$ at the wave-vector $\mathbf{Q}=(0.69,1,0)$ [19]. In this compound, the spin dynamics is basically governed by the Kondo relaxation. However it is not the only ingredient and the exchange interaction must be taken into account for example using the RPA approximation ${ }^{2}$. Due to the temperature factor, there is non-zero intensity for neutron energy gain $(E<0)$ only at high temperatures when excitations are thermally activated in the system. The right panels shows the corresponding imaginary part of the susceptibility. This quantity has a maximum as a function of energy even if there is no pole in Eq. (5.5). This is related to the fact that $\chi^{\prime \prime}$ is an odd function of energy. This position in energy of the maximum corresponds to $\Gamma_{K}$ in Eq. (5.5). Here we obtained $\Gamma_{K} \approx 1 \mathrm{meV}$ at $2.5 \mathrm{~K}$ which is consistent with the known Kondo temperature of this system.

${ }^{2}$ When taking the interaction $J_{\mathbf{q}}$ into account, the susceptibility becomes $\chi^{\prime \prime}(\mathbf{q}, \omega)=\frac{\chi_{\mathbf{q}}^{\prime} \Gamma_{\mathbf{q}}}{\omega^{2}+\Gamma_{\mathbf{q}}^{2}}$ with $\chi_{\mathbf{q}}^{\prime}=\frac{\chi_{K}}{1-\chi_{K} J \mathbf{q}}$ and $\Gamma_{\mathbf{q}}=\Gamma_{K}\left(1-\chi_{K} J_{\mathbf{q}}\right)$. 

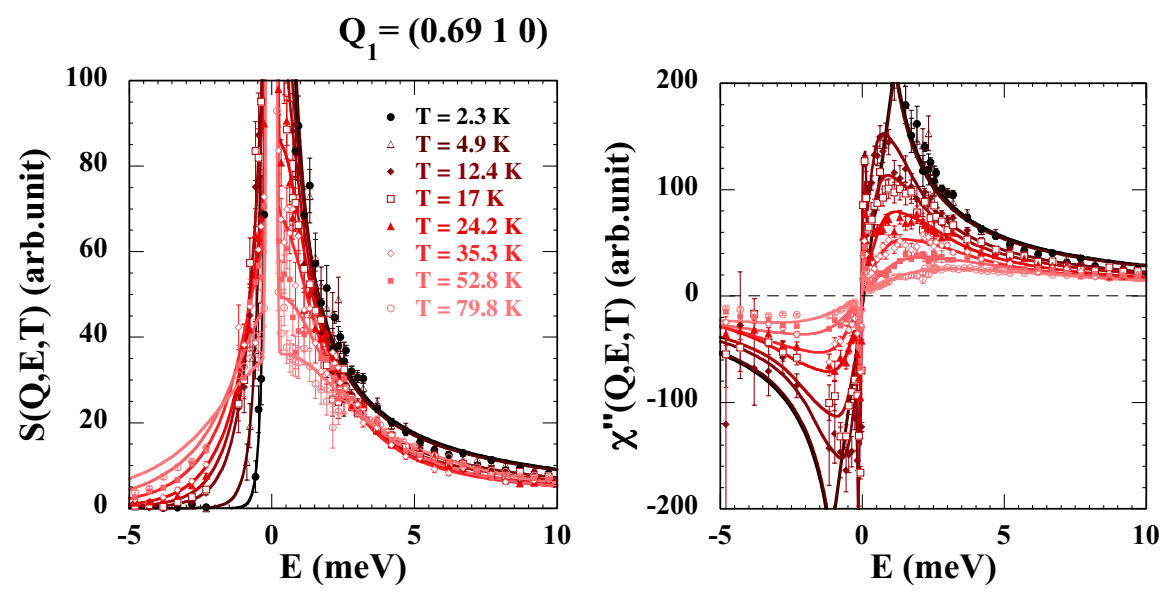

Figure 8. Scattering function and imaginary part of the dynamical spin susceptibility measured in $\mathrm{CeRu}_{2} \mathrm{Si}_{2}$ at $\mathbf{Q}=(0.69,1,0)[19]$.

\section{EXCITATIONS IN ITINERANT ELECTRON SYSTEMS}

Up to now localized spins described by the Heisenberg Hamiltonian were considered. For itinerant electron systems, a band description is more appropriate. One of the simplest model used to describe itinerant systems is the Hubbard model that contains a hopping term $t_{i, j}$ from one site $i$ to another site $j$ and a local repulsion term $U$ acting on spins with different orientation (Pauli principle excludes spins having the same state):

$$
H_{\text {Hubbard }}=-\sum_{i, j} t_{i, j, \sigma} c_{i \sigma}^{\dagger} c_{j, \sigma}+\sum_{i} U n_{i, \uparrow} n_{i, \downarrow}
$$

Without interaction $U=0$, the dynamical spin susceptibility is described by the Lindhard function:

$$
\chi_{0}(\mathbf{q}, \omega)=-2 \mu_{B}^{2} \sum_{\mathbf{k}} \frac{f_{\mathbf{k}+\mathbf{q}, \sigma^{\prime}}-f_{\mathbf{k}, \sigma}}{\epsilon_{\mathbf{k}+\mathbf{q}, \sigma^{\prime}}-\epsilon_{\mathbf{k}, \sigma}-\hbar \omega+i \epsilon}
$$

where $f_{\mathbf{k}, \sigma}$ is the Fermi function and $\epsilon_{\mathbf{k}, \sigma}$ is the electronic band energy for spin $\sigma$. Such a susceptibility gives rise to a continuum of excitations and each excitation of wave-vector $\mathbf{k}$ consists in creating a hole at an energy $\epsilon_{\mathbf{k}}$ below the Fermi energy $\epsilon_{F}$ and an electron with energy $\epsilon_{\mathbf{k}+\mathbf{q}}$ above the Fermi energy. This is shown in Figure 9a. When the interaction $U$ is taken into account, there is a splitting of the spin up and spin down bands: $\Delta=U\left(n_{u p}-n_{\text {down }}\right)$ and ferromagnetism occurs when the Stoner criterion is reached, $U n\left(\epsilon_{F}\right)>1$ where $n\left(\epsilon_{F}\right)$ is the density of state at the Fermi level. The dynamical spin susceptibility must be separated into an intraband term (longitudinal part, $\chi^{z z}$ ) giving rise to individual excitations as in the case $U=0$ and an interband term (transverse susceptibility, $\chi^{+-}$) consisting of individual excitations and a collective spin wave mode. This last part is depicted in Figure 9b. The spin wave excitations merge into the Stoner continuum at high wave-vector. To get an accurate description of a given system with a model of itinerant electron magnetism, one must take into account the complexity of $\epsilon_{\mathbf{k}}$. Whatever this complexity, the salient features of this kind of model are the presence of continuum of excitations and the importance of Fermi surface topology. Figure 9c shows the excitation spectrum measured in the nearly ferromagnetic (also named exchange enhanced paramagnetic) compound $\mathrm{Ni}_{3} \mathrm{Ga}$ [20]. The spectrum is broad in energy and wave-vector (here data are collected as a function of scattering angle on a polycristalline sample). At first sight this can be related either to a model of localized spins above $T_{\text {Curie }}$ (Sect. 5) or a model of itinerant electron magnetism (Sect. 6). However, the relaxation rate 
a)

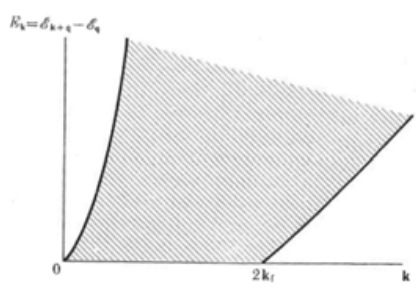

b)

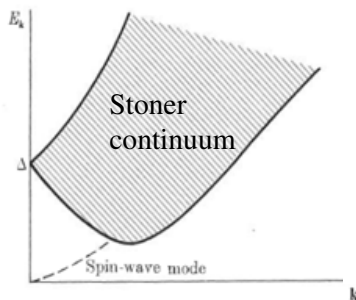

c)

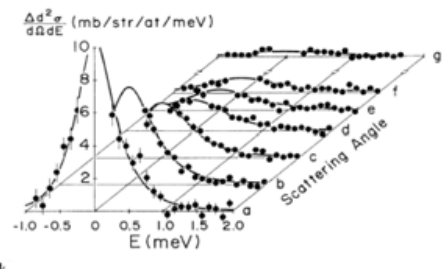

Figure 9. a) Energy spectrum of electron-hole excitation in a metal. b) Energy spectrum for interband transition in a ferromagnetic itinerant metal for $U>\epsilon_{F}$. c) Fluctuation spectrum of $\mathrm{Ni}_{3} \mathrm{Ga}$ [20].
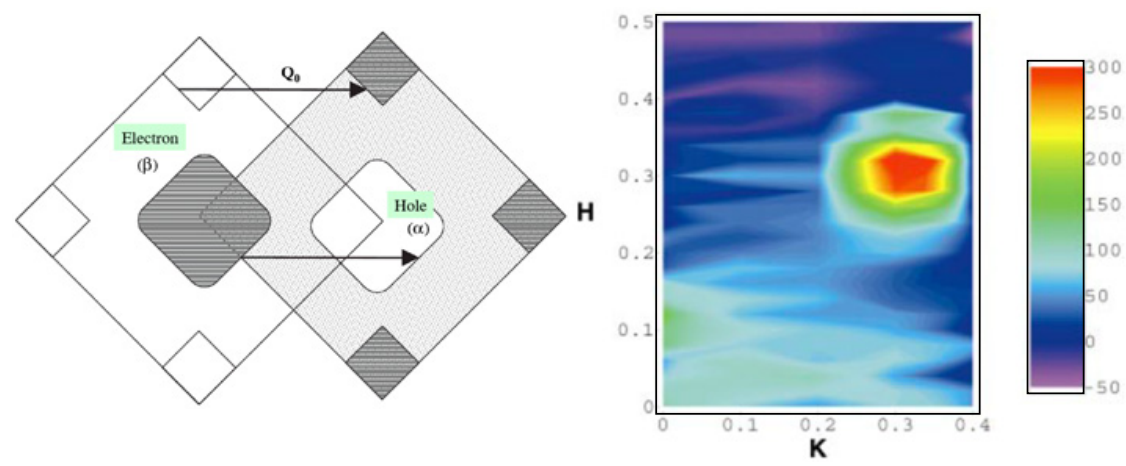

Figure 10. Left: sketch of the nesting feature between $\alpha$ and $\beta$ bands of $\operatorname{Sr}_{2} \mathrm{RuO}_{4}$. Right: $(H, K)$ map of the magnetic excitations in $\mathrm{Sr}_{2} \mathrm{RuO}_{4}$ at $10 \mathrm{~K}$ for an energy transfer of $4.1 \mathrm{meV}$ [21].

is established to be $\Gamma_{q}=\gamma q \chi^{-1}(q)$ and this linear $q$ dependence at low $q$ constitutes a landmark of Fermi liquid theory.

Another typical feature of itinerant electron physics is the role of the Fermi surface topology and this is illustrated in the compound $\mathrm{Sr}_{2} \mathrm{RuO}_{4}$. The $\mathrm{Ru}^{4+}$ ion has the configuration $4 d^{4}$ with a spin $S=1$ on the $t_{2 g}$ crystal field ground state. The Fermi surface has a hole band $\alpha$ and an electron band $\beta$ coming from the orbitals $d_{y z}$ and $d_{z x}$ for which nesting properties occurs for $\mathbf{Q}_{0}=(1 / 3,1 / 3,0)$, i.e. $\epsilon_{\alpha}(\mathbf{k}) \approx \epsilon_{\beta}\left(\mathbf{k}+\mathbf{Q}_{\mathbf{0}}\right)$. The susceptibility given by the Lindhard formula (6.2) is therefore enhanced for $\mathbf{Q}_{\mathbf{0}}$ since many electronic states characterized by $\mathbf{k}$ follows $\epsilon_{\alpha}(\mathbf{k}) \approx \epsilon_{\beta}\left(\mathbf{k}+\mathbf{Q}_{\mathbf{0}}\right)$. A ferromagnetic interaction must be included in order to describe the system and this can be done by using the RPA Eq. (4.1) taking the Lindhard susceptibility as the non-interacting part and the adequate ferromagnetic exchange $J_{\mathbf{q}}$. Figure 10 shows a sketch of the nesting feature and the excitation spectrum of $\mathrm{Sr}_{2} \mathrm{RuO}_{4}$ measured in the basal plane of the tetragonal structure for a constant energy of $4.1 \mathrm{meV}$ [21]. The most intense part of the spectrum corresponds to the incommensurate fluctuations related to the nesting features while an increase of fluctuations is also observed near $q=0$ and this corresponds to ferromagnetic fluctuations arising from exchange interaction.

An important topic is the study of magnetic excitations in superconductors and the possible feedback effect of superconductivity on the excitation spectrum. This topic is developped by Y. Sidis in the present book. In the superconducting phase, the electronic excitation spectrum acquires a gap $\Delta_{\mathbf{k}}$ and the quasiparticle dispersion relation is $E_{k}=\sqrt{\Delta_{\mathbf{k}}^{2}+\epsilon_{\mathbf{k}}^{2}}$. The corresponding Lindhard function is modified 


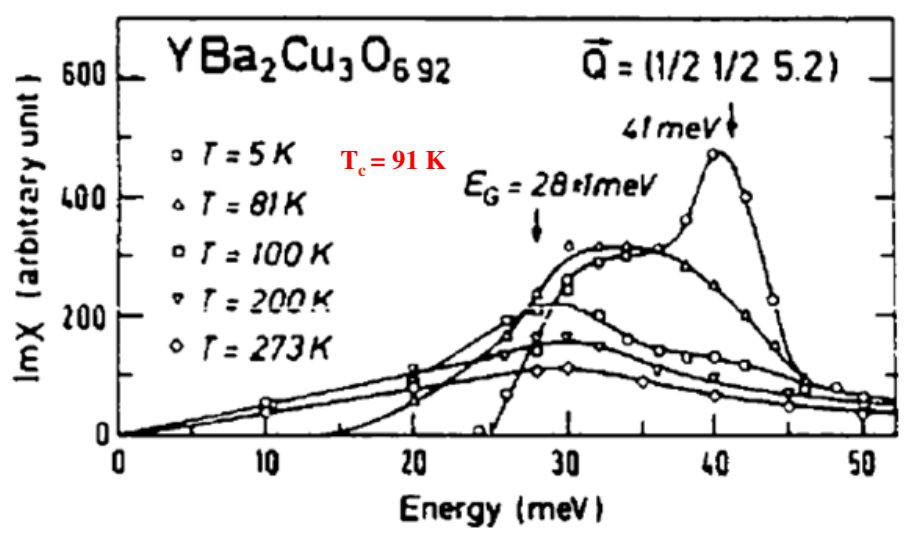

Figure 11. Energy spectrum at the antiferromagnetic wave-vector $(1 / 2,1 / 2,5 / 2)$ of the unconventional superconductor $\mathrm{YBa}_{2} \mathrm{Cu}_{3} \mathrm{O}_{6.92}$ for several temperatures below and above $T_{c}$ [22].

by a coherence factor specific of the superconducting state:

$$
\chi_{0}(\mathbf{q}, \omega)=-2 \mu_{B}^{2} \sum_{\mathbf{k}}\left(1-\frac{\Delta_{\mathbf{k}} \Delta_{\mathbf{k}+\mathbf{q}}+\varepsilon_{\mathbf{k}} \varepsilon_{\mathbf{k}+\mathbf{q}}}{E_{\mathbf{k}} E_{\mathbf{k}+\mathbf{q}}}\right) \frac{f_{\mathbf{k}+\mathbf{q}, \sigma^{\prime}}+f_{\mathbf{k}, \sigma}-1}{E_{\mathbf{k}+\mathbf{q}, \sigma^{\prime}}-E_{\mathbf{k}, \sigma}-\hbar \omega+i \epsilon} .
$$

Due to the coherence factor, the magnetic response is enhanced if the superconducting gap changes sign at a specific wave-vector $\mathbf{Q}: \Delta_{\mathbf{k}}=-\Delta_{\mathbf{k}+\mathbf{Q}}$. This is the case for unconventional superconductors like cuprates and heavy fermion systems having a superconducting gap following the symmetry $d_{x^{2}-y^{2}}$. If interactions are taken into account in a RPA approximation, a pole can occur in the interacting susceptibility. This new mode appears below the superconducting gap and is often called resonance or spin exciton: it is an $S=1$ particule-hole excitation bound at an energy below the pair-breaking one. Such a mode discovered in $\mathrm{YBa}_{2} \mathrm{Cu}_{3} \mathrm{O}_{6.92}$ is shown in Figure 11 . Above $T_{c}=91 \mathrm{~K}$, the spectrum is gapless with a continuum of state characteristic of a paramagnetic metal. Below $T_{c}$, a gap opens at the antiferromagnetic wavevector and a resonance peak appears at $41 \mathrm{meV}$ which corresponds to $5.2 k_{B} T_{c}$. In compounds where such a resonance peak is observed, it is believed that magnetic fluctuations are responsible for the pairing of electrons in the superconducting phase (in contrast to phonon mediated pairing for more conventional superconductors).

\section{QUANTUM EFFECTS, LOW DIMENSION AND FRUSTRATION}

Up to now, classical spins on a three dimensional (3D) lattice were considered. The concept of large spins allows for example to make spin wave approximation that is to consider small deviations from an ordered moment. A three dimensional lattice means that spins are surrounded by several equivalent neighbors and that mean-field methods are likely to be pertinent (like the RPA approximation) except when frustration arises (see below). In contrast ordering does not occurs in low dimensions: The Mermin-Wagner theorem states that there is no order at finite temperature in 1D and 2D, for systems with continuous broken symmetry. However real systems are often three dimensional due to sizable interchain or inter-plane coupling leading to dimensional crossover as a function of temperature. One of the best highlight of quantum effects is provided by the physics of antiferromagnetic quantum spin chains. The magnetic excitation spectrum is completely different for integer or half-integer spins. Figure 12 shows the excitation spectrum of the $S=1 / 2$ antiferromagnetic chain compound $\mathrm{KCuF}_{3}$ measured using single crystal sample on the TOF machine MAPS at ISIS taking benefit from the 1D nature of the system [23]. The low-lying excitation spectrum is formed of a continuum of states located between the 


\section{Collection SFN}
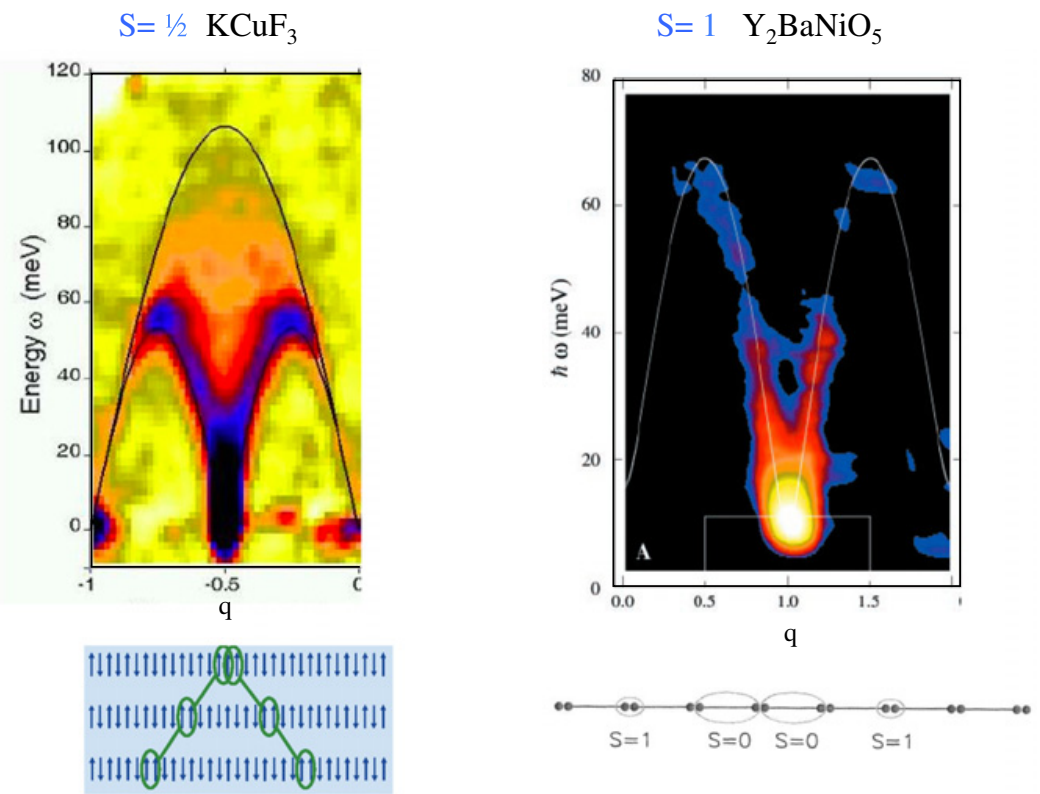

Figure 12. Left: continuum of spinon excitations for the $S=1 / 2$ antiferromagnetic spin chain compound $\mathrm{KCuF}_{3}$ [23]. The lower panel shows a picture of spinons propagation in a chain for three different times. Right: excitation spectrum of the Haldane chain compound ( $S=1$ antiferromagnetic chain) $\mathrm{Y}_{2} \mathrm{BaNiO}_{5}$ and picture of the corresponding AKTL state [24].

two black curves and corresponds to excitations of pairs of so-called spinons depicted at the bottom of the figure: they are domain wall like quasiparticles separating antiferromagnetic domains. In contrast, the excitation spectrum for the $S=1$ chain compound $\mathrm{Y}_{2} \mathrm{BaNiO}_{5}$ shows single particle excitations with the peculiar feature that a gap opens at the antiferromagnetic wave-vector. This behaviour was theoretically predicted by Haldane before being confirmed by experiment. The ground state of such system is a singlet and the first excited state a triplet excitation with a gap $\Delta \propto \exp (-\pi S)$. From this latter formula, it is clear that the gap vanishes when the size of the spin increases and this underlines the quantum nature of the state. The lower frame of the panel depicts the Haldane chain in term of a valence bound state provided by the AKLT model (Affleck, Kennedy, Lieb and Tagaki) where each spin 1 is decomposed into two spins $1 / 2$ combined between neighbors.

Beyond spin chains, many studies are also devoted to spin ladders having 1, 2, n...legs and to 2D systems. For 3D systems, an interesting case treated in the present book by T. Fennel is the one of geometrical frustration. For certain geometries (triangles, tetrahedra), all interactions between neighbors cannot be satisfied. This gives rise to degenerate ground states and specific spin fluctuation spectrum with low energy magnetic excitations

\section{POLARIZED INELASTIC NEUTRON SCATTERING}

After having reviewed magnetic excitations, we go back to some instrumental aspects related to the possibility to polarize the neutron beam i.e to use a beam with a well defined neutron spin state on the incident and/or scattered beam. The general scattering cross-sections for polarized neutrons are given by the Blume-Maleyev equations. They are discussed in this book by M. Enderle and E. Ressouche and the inelastic polarized magnetic scattering was described in great details by L.P. Regnault in the JDN13 

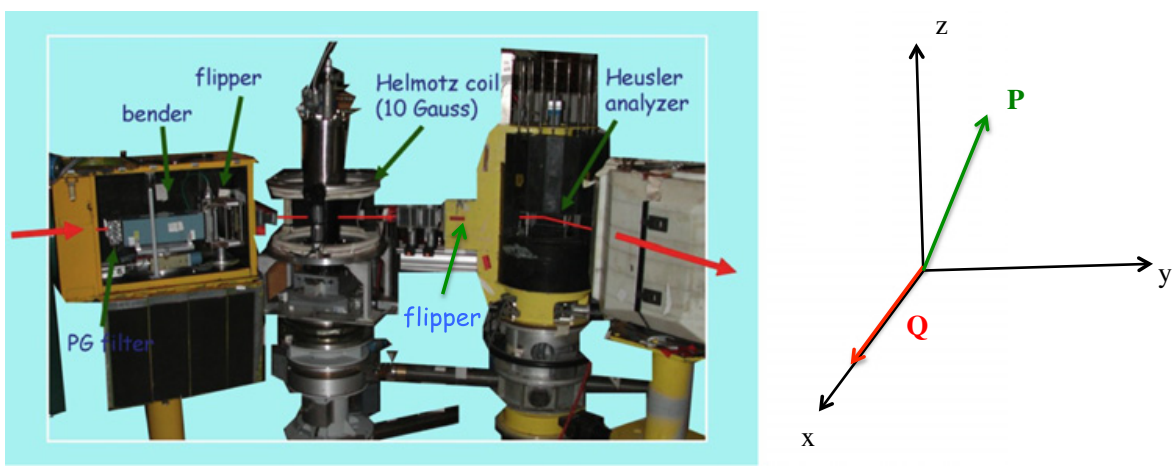

Figure 13. Experimental setup used for longitudinal polarimetry on the cold TAS 4F1 at LLB and axis conventions used for describing the scattering.

lecture book [25]. If we call $\mathbf{P}_{\mathbf{i}}$ the initial polarization of the beam and $\mathbf{P}_{\mathbf{f}}$ the final one, the two BlumeMaleyev equations give the scattered intensity as a function of $\mathbf{P}_{\mathbf{i}}$ and the polarization $\mathbf{P}_{\mathbf{f}}$ as a function of $\mathbf{P}_{\mathbf{i}}$. Three experimental setups are mostly used:

- "half polarized" experiment: either $\mathbf{P}_{\mathbf{i}}$ or $\mathbf{P}_{\mathbf{f}}$ is defined, the other one being zero.

- longitudinal polarimetry: this gives access to the projection of $\mathbf{P}_{\mathbf{f}}$ on $\mathbf{P}_{\mathbf{i}}$.

- spherical polarimetry: $\mathbf{P}_{\mathbf{i}}$ and $\mathbf{P}_{\mathbf{f}}$ are independently determined.

Most of the polarized INS experiments are performed on TAS (with a few exceptions like D16 at ILL). In this section, we focus first on the more traditional longitudinal polarization method; other experimental setups are given in the lecture by M. Enderle. A typical longitudinal polarization setup on a TAS is shown in Figure 13: The initial beam is polarized by a bender (supermirror polarizing device) placed after the graphite monochromator (it can be another kind of polarizer: Heusler monochromator, ${ }^{3} \mathrm{He}$ spin filter), a flipper is used to reverse the polarization, an Helmholtz coil allows to define the polarization on the sample. Then a combination of a flipper and an Heusler analyzer are used to analyze the final polarization. Guide fields are present along the neutron path in order to keep the polarization. The convention shown in Figure 13 is to define $x / / \mathbf{Q}, y \perp \mathbf{Q}$ in the scattering plane and $z$ vertical and $\mathbf{P}$ is defined by the choice of currents in the Helmholtz coil. Usually it is chosen along $x, y$ or $z$. For simplicity, we neglect nuclear spin incoherent scattering. We also neglect nuclear-magnetic interference terms and chiral terms. In such a case $\mathbf{P}_{\mathbf{f}}$ is either parallel to $\mathbf{P}_{\mathbf{i}}$ (the so-called Non Spin Flip (NSF) scattering) or anti-parallel to $\mathbf{P}_{\mathbf{i}}$ (the so-called Spin Flip (SF) scattering). The different cross-sections are:

$$
\begin{aligned}
I_{x}^{N S F} & \propto N \\
I_{x}^{S F} & \propto<S_{Q}^{y} S_{-Q}^{y}>_{\omega}+<S_{Q}^{z} S_{-Q}^{z}>_{\omega} \\
I_{y}^{N S F} & \propto N+<S_{Q}^{y} S_{-Q}^{y}>_{\omega} \\
I_{y}^{S F} & \propto<S_{Q}^{z} S_{-Q}^{z}>_{\omega} \\
I_{z}^{N S F} & \propto N+<S_{Q}^{z} S_{-Q}^{z}>_{\omega} \\
I_{z}^{S F} & \propto<S_{Q}^{y} S_{-Q}^{y}>_{\omega}
\end{aligned}
$$

$N$ is the nuclear scattering (without taking into account nuclear spin) and $<S_{Q}^{\alpha} S_{-Q}^{\alpha}>_{\omega}$ is a short notation for the space and time Fourier transform of the spin-spin correlation function. The important 


\section{Collection SFN}
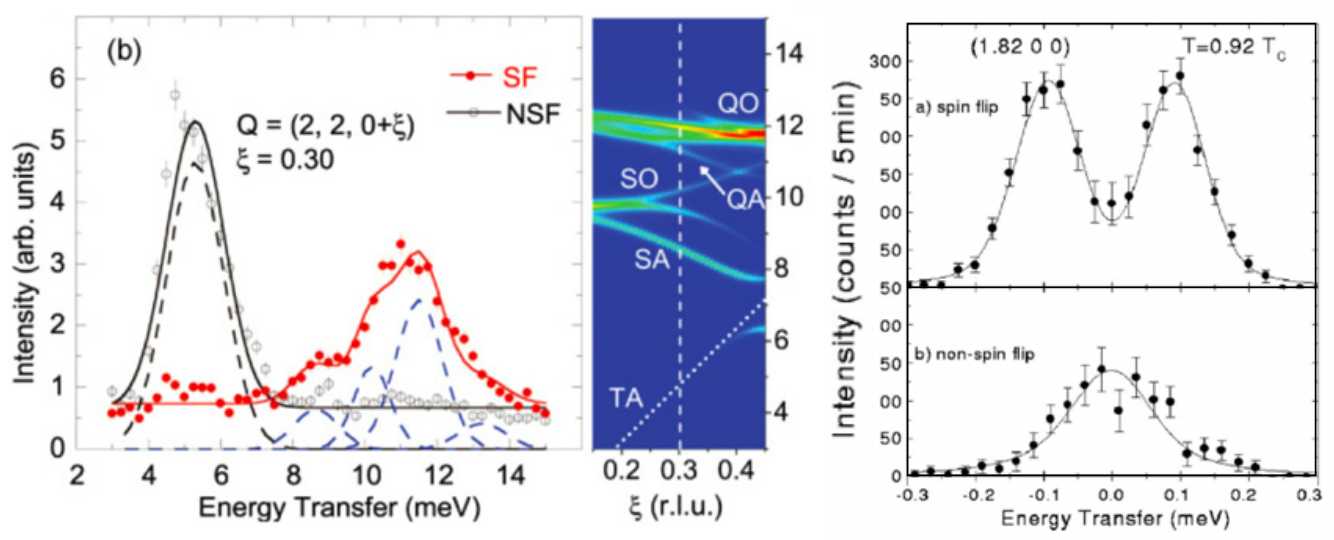

Figure 14. Left: energy spectrum of the $\mathrm{AF}$ compound $\mathrm{UO}_{2}\left(T_{N}=30.8 \mathrm{~K}\right)$ at $T=2 \mathrm{~K}$ for $\mathbf{Q}=(2,2,0.3)$ in the SF and NSF channels for $\mathbf{P} / / \mathbf{Q}$ and corresponding dispersion relation of TA phonon and magnetic excitations (spin waves (SA, SO) and "quadrupolar" modes (QA, QO)) [26]. Right: SF, NSF neutron energy spectra measured at $\mathbf{Q}=(1.82,0,0)$ and $T=0.92 T_{\text {Curie }}$ in the ferromagnet EuS [27].

features are:

- nuclear scattering is NSF (except for nuclear spin incoherent scattering neglected here).

- spin flip occurs for the magnetic components perpendicular to $\mathbf{P}$.

- non spin flip occurs for the magnetic components parallel to $\mathbf{P}$.

- one can obtain individual $<S_{Q}^{\alpha} S_{-Q}^{\alpha}>_{\omega}$ by performing subtraction of different terms in Eq. (8).

The left panel of Figure 14 illustrates the separation of phonon and magnon excitations in $\mathrm{UO}_{2}$ by using polarized neutron [26]. In this example, $\mathbf{P} / / \mathbf{Q}$ and all the magnetism appears in the SF channel. The peak at around $5 \mathrm{meV}$ in the NSF channel is a transverse acoustic phonon while several magnetic modes are measured in the SF channel. The dispersion curves of these excitations are shown on the right with a dash line indicating the peculiar $\mathbf{Q}$ vector for which the energy scan is performed. The second example shown in the right panel of Figure 14 concerns the ferromagnet EuS. For $\mathbf{P} / / \mathbf{z}$ and the ferromagnetic moments also along z-axis, the spin wave excitations appear in the SF channel since the spin deviation occurs perpendicular to the magnetization (upper frame). Supplementary excitations appear in the NSF channel (middle); they are therefore polarized along the magnetic moment and corresponds to longitudinal fluctuations (fluctuation of the ordered moment size). These quasielastic fluctuations are the ones described in Section 5; they occurs here in an ordered phase in coexistence with the spin wave modes.

Very often the chiral contribution which is the cross product between two spin components cannot be neglected. With the axis defined above, the chiral correlation function is $M_{c h}=i\left(<S_{Q}^{y} S_{-Q}^{z}>-<\right.$ $S_{Q}^{z} S_{-Q}^{y}>$ ). The only modification arises for $\mathbf{P} / / \mathbf{Q}$, among SF scattering, one must distinguish between +- and -+ cross-sections and (8.2) becomes:

$$
\begin{aligned}
& I_{x}^{+-} \propto<S_{Q}^{y} S_{-Q}^{y}>_{\omega}+<S_{Q}^{z} S_{-Q}^{z}>_{\omega}-M_{c h} \\
& I_{x}^{-+} \propto<S_{Q}^{y} S_{-Q}^{y}>_{\omega}+<S_{Q}^{z} S_{-Q}^{z}>_{\omega}+M_{c h} .
\end{aligned}
$$

A very current experimental setup is to use an horizontal field magnet instead of the Helmholz coil for a geometry with $\mathbf{P} / / \mathbf{Q}$ and to use half-polarized setup, for example with an incident beam polarized and 


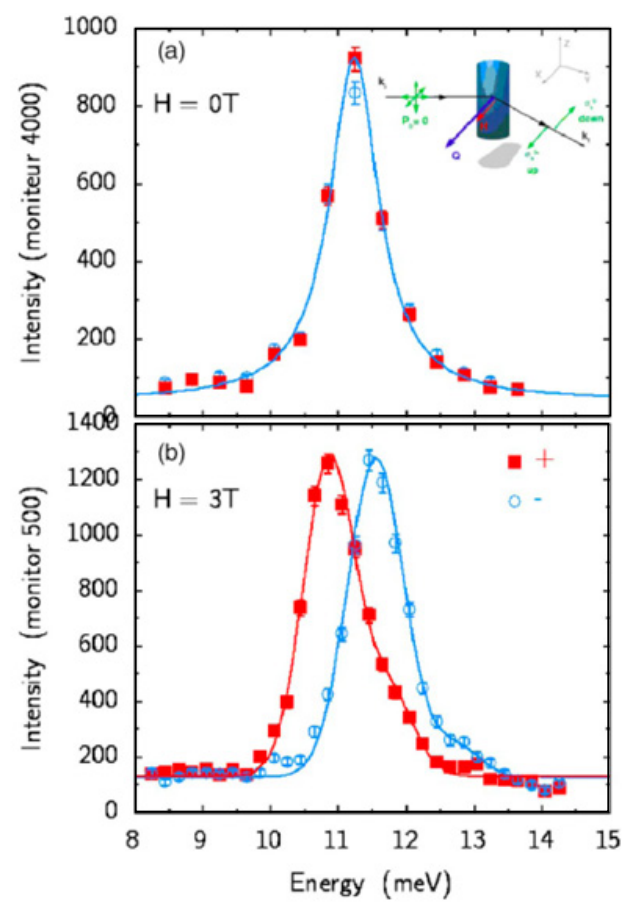

Figure 15. Inelastic spectra at $H=0 \mathrm{~T}$ (a) and $H=3 \mathrm{~T}$ (b) of $\mathrm{Sr}_{14} \mathrm{Cu}_{24} \mathrm{O}_{41}$ at $\mathbf{Q}=(2.5,0,0.25)$ measured with half polarized setup [28]. Blue and red symbols corresponds to different polarization channels.

without polarization analysis. The previous sets of equations give the corresponding scattering crosssections:

$$
\begin{aligned}
& I_{x}^{+0} \propto N+<S_{Q}^{y} S_{-Q}^{y}>_{\omega}+<S_{Q}^{z} S_{-Q}^{z}>_{\omega}-M_{c h} \\
& I_{x}^{-0} \propto N+<S_{Q}^{y} S_{-Q}^{y}>_{\omega}+<S_{Q}^{z} S_{-Q}^{z}>_{\omega}+M_{c h} .
\end{aligned}
$$

What is of interest here is to benefit from the contrast provided by the chiral term in Eqs. (8.9)-(8.10). This is illustrated by an experiment carried out on the spin ladder compound $\mathrm{Sr}_{14} \mathrm{Cu}_{24} \mathrm{O}_{41}$ shown in figure 15 [28]. A singlet-triplet excitation is characteristic of the chain subsystem of this compound ( $\mathrm{S}=1 / 2$ spins form dimers along the chain in this compound). The corresponding gap $\Delta \approx 11 \mathrm{meV}$ is shown in the spectrum at $\mathrm{H}=0 \mathrm{~T}$. Under a magnetic field of $3 \mathrm{~T}$, the triplet splits into three components labelled by the quantum number $S_{z}$. The mode with $S_{z}=0$ is polarized along $\mathbf{Q}$ and cannot be observed by INS (since only fluctuations perpendicular to $\mathbf{Q}$ can be seen). The $S_{z}=-1\left(S_{z}=1\right)$ mode of the triplet is observed at the energies $\Delta-\mu H(\Delta+\mu H)$ in the polarization channel $I^{+0}\left(I^{-0}\right)$. Hence, the modes at different energies $\Delta \pm \mu H$ contribute separately to different polarization channels (red and blue lines in Fig. 15 bottom). This is related to the fact that the two modes have opposite handedness. Without polarization, the two peaks will overlap and the splitting, which is much smaller than $\Delta$, will not be resolved.

\section{CONCLUSION}

Inelastic Neutron Scattering is a unique tool to measure various kinds of spin dynamics from local to collective excitations in a wide range of $(\mathbf{Q}, \omega)$ space. The measured scattering function can be 


\section{Collection SFN}

easily related to a microscopic description of the studied system using either matrix elements, spin-spin correlation functions or the dynamical spin susceptibility formalism. Finally polarized Inelastic Neutron Scattering allows not only to unambiguously show the magnetic nature of the measured spectrum but also to gain more insight into detailed characteristics of the magnetic excitation.

\section{Acknowledgments}

We acknowledge S. Petit for a careful reading of the manuscript and helpful comments.

\section{References}

[1] Magnetic Excitations, W.G. Stirling et K.A. McEwen, Methods of Experimental Physics Vol. 23 Part C Academic Press (1987).

[2] A. Furrer, J. Mesot and T. Strässle, Neutron Scattering in Condensed Matter Physics, World Scientific (2009).

[3] G.L. Squires, Introduction to the theory of thermal neutron scattering, Cambridge University Press (1978).

[4] S.W. Lovesey, Theory of Neutron Scattering from Condensed Matter, Volume 2, Oxford Science Publications (1984).

[5] Y.A. Izyumov and N.A. Chernoplekov, Neutron Spectroscopy, Plenum (1994).

[6] Neutron and X-ray Spectroscopy, F. Hippert, E. Geissler, J.L Hodeau, E. Lelièvre-Berna and J.-R. Regnard Eds. Springer (2005).

[7] G. Shirane, S.M. Shapiro and J. Tranquada, Neutron Scattering with a Triple-Axis Spectrometer, Basic Techniques, Cambridge University Press (2002).

[8] B. Hennion, La diffusion inélastique des neutrons sur monocristal. Le spectromètre 3-axe., JDN 16, EDP Sciences.

[9] J. Ollivier and J.-M. Zanotti, La diffusion inélastique de neutrons par temps de vol, JDN 16, EDP Sciences.

[10] A. Villaume et al., Phys. Rev. B 78 (2008) 012504.

[11] A.D. Hillier et al., Phys. Rev. B 85 (2012) 134405.

[12] A. Furrer and H.G. Purwins, J. Phys. C: Solid State Phys. 9 (1976) 1491.

[13] Y.-J. Kim et al., Phys. Rev. B 84 (2011) 085132.

[14] S. Petit, Les ondes de spin, JDN 16, EDP Sciences.

[15] T. Chatterji, Neutron Scattering from Magnetic Materials, Elsevier (2006) p 268.

[16] L.P. Regnault et al., J. Magn. Magn. Mat. 31-34 (1983) 1205.

[17] K. Kuwahara et al., Phys. Rev. Lett. 95 (2005) 107003.

[18] A. Huxley, S. Raymond and E. Ressouche, Phys. Rev. Lett. 91 (2003) 207201.

[19] W. Knafo et al., Phys. Rev. B. 70 (2004) 174401.

[20] N. Bernhoeft et al., Phys. Rev. Lett. 62 (1989) 657.

[21] M. Braden et al., Phys. Rev. B 66 (2002) 064522.

[22] J. Rossat-Mignot et al., Physica C 185-189 (1991) 86.

[23] D.A. Tennant et al., Phys. Rev. B 70 (1993) 4003.

[24] G. Xu et al., Science 289 (2000) 419.

[25] L.P. Regnault, Polarimétrie neutronique longitudinale et sphérique en diffusion inélastique, JDN 13, EDP Sciences.

[26] R. Caciuffo et al., Phys. Rev. B 84 (2011) 104409.

[27] P. Böni et al., Phys. Rev. B 65 (2002) 14434.

[28] E. Lorenzo et al., Phys. Rev. B 75 (2007) 054418. 